If the rank of the jacobian matrix is $n-2$, that is, if there exist two relations (and not more) between the $f$ 's which do not involve the $x$ 's, the hypersurfaces will all have the origin as a double point, and terms of the second degree of (10) tell us the nature of the singular point.

We note that equation (7) would be satisfied if every element vanished. This would lead to $n^{2}$ linear equations in the quantities $f_{k}{ }^{\prime}$. It is known that if the algebra is commutative and associative, these $n^{2}$ equations have a unique solution for all analytic functions of $w$. The derivative congruence would in this case degenerate into a congruence of points, the function being monogenic.

UNIVERSITY OF ILLINOIS

\title{
ON FINITE AND INFINITE COMPLETELY MONOTONIC SEQUENCES*
}

\section{BY I. J. SCHOENBERG}

1. Introduction. As one of several important results concerning the problem of moments for a finite interval, Hausdorff $\dagger$ has proved the following theorem.

The problem of moments, which is to find a real function $\chi(t)$ for $0 \leqq t \leqq 1$, such that

$$
\mu_{n}=\int_{0}^{1} t^{n} d \chi(t), \quad(n=0,1,2, \cdots),
$$

in which the sequence $\mu_{0}, \mu_{1}, \mu_{2}, \cdots$ is given in advance, has a monotonic non-decreasing solution, if and only if

(2) $\Delta^{l} \mu_{m}=\sum_{\nu=0}^{l}(-1)^{\nu}\left(\begin{array}{l}l \\ \nu\end{array}\right) \mu_{m+\nu} \geqq 0, \quad$ for $l, m=0,1,2, \cdots$,

in which case the sequence is said to be completely monotonic.

The same problem (1) has a solution $\chi(t)$ which is of bounded variation, if and only if

* Presented to the Society, November 28, 1931.

$\dagger$ Ueber das Momentenproblem für ein endliches Interval, Mathematische Zeitschrift, vol. 16 (1923), pp. $220-248$. We refer to $\$ \S 1$ and 2 . 


$$
\sum_{m=0}^{p}\left(\begin{array}{c}
p \\
m
\end{array}\right)\left|\Delta^{p-m_{\mu}} \mu_{m}\right|=O(1) \text { for } p \rightarrow \infty .^{*}
$$

Hausdorff proves this theorem starting from the system (1), which defines a positive linear functional operation by interpolation in function space, and proves that the conditions (2) are necessary and sufficient for the existence of such a functional operation defined by the function $\chi(t)$. In this note we shall proceed in the opposite way. We shall solve the set (2) of linear inequalities in infinitely many variables and shall find that its most general solution may be represented parametrically in the form (1). In another paper the same point of view will be applied to similar but more general problems considered by Carathéodory, F. Riesz, and Hausdorff.

2. Finite Sequences. It seems natural to start first with the following problem. Let us call the finite sequence $\mu_{0}, \mu_{1}, \cdots, \mu_{p}$ completely monotonic if

(4) $\Delta^{l} \mu_{m} \geqq 0$ for $l, m=0,1,2, \cdots, p$, with $l+m \leqq p . \dagger$

We seek a parametric representation for the most general solution of the set (4) of linear inequalities.

Such a representation is readily found. The identity $\Delta^{l} \mu_{m}$ $=\Delta^{l} \mu_{m+1}+\Delta^{l+1} \mu_{m}$ shows that the set (4) is a consequence of its sub-set

$$
\Delta^{p-m} \mu_{m} \geqq 0, \quad \text { for } m=0,1,2, \cdots, p,
$$

which means that the $p+1$ linear forms

$$
\begin{array}{rlr}
\rho_{p, 0} & =\mu_{0}-\left(\begin{array}{l}
p \\
1
\end{array}\right) \mu_{1}+\left(\begin{array}{c}
p \\
2
\end{array}\right) & \mu_{2}-\cdots+(-1)^{p} \mu_{p}, \\
\rho_{p, 1} & = & \mu_{1}-\left(\begin{array}{c}
p-1 \\
1
\end{array}\right) \mu_{2}+\cdots+(-1)^{p-1} \mu_{p}, \\
\rho_{p, 2} & = & \mu_{2}-\cdots+(-1)^{p-2} \mu_{p}, \\
(6) & \cdot & \vdots \\
\rho_{p, p-1}= & \cdot & \mu_{p-1}-\mu_{p}, \\
\rho_{p, p}= & \mu_{p},
\end{array}
$$

* This statement is certainly verified for the previous case, because (2) gives $\sum_{m=0}^{p}\left(\begin{array}{c}p \\ m\end{array}\right)\left|\Delta^{p-m_{m}} \mu_{m}\right|=\sum_{m-0}^{p}\left(\begin{array}{c}p \\ m\end{array}\right) \Delta^{p-m_{\mu}} \mu_{m}=\mu_{0}$.

$\dagger$ This definition is justified by the fact that these are all the higher differences which may be derived from our finite sequence. 
should be non-negative. The linear transformation inverse to (6) is

$$
\begin{aligned}
& \mu_{0}=\rho_{p, 0}+\left(\begin{array}{l}
p \\
1
\end{array}\right) \rho_{p, 1}+\left(\begin{array}{l}
p \\
2
\end{array}\right) \quad \rho_{p, 2}+\cdots+\rho_{p, p} \\
& \mu_{1}=\quad \rho_{p, 1}+\left(\begin{array}{c}
p-1 \\
1
\end{array}\right) \rho_{p, 2}+\cdots+\rho_{p, p}, \\
& \text { (7) } \mu_{2}= \\
& : \\
& \mu_{p-1}= \\
& \mu_{p}= \\
& \rho_{p, 2}+\cdots+\rho_{p, p}, \\
& \rho_{p, p-1}+\rho_{p, p}, \\
& \rho_{p, p} .
\end{aligned}
$$

Our problem is solved by the set (7) which yields for

$$
\rho_{p, 0} \geqq 0, \rho_{p, 1} \geqq 0, \cdots, \rho_{p, p} \geqq 0,
$$

a parametric representation for the most general finite sequence $\mu_{0}, \mu_{1}, \cdots, \mu_{p}$, which is completely monotonic.

3. Infinite Sequences. We return to our original problem. The inequalities (2) are certainly necessary for a parametric representation (1) with $\chi(t)$ monotonic, since

$$
\Delta^{l} \mu_{m}=\int_{0}^{1} t^{m}(1-t)^{l} d \chi(t) \geqq 0 .
$$

Conversely, suppose that now the inequalities (2) hold. The set of inequalities (4) holds for every value of $p=1,2,3, \cdots$ and therefore also (6), (7), and (8) are true for these values. We shall obtain the set of equations (1) from the set (7) by making $p \rightarrow \infty$.

The $n$th equation of the set ( 7$)$ gives for $p \geqq n$

$$
\mu_{n}=\sum_{m=n}^{p}\left(\begin{array}{c}
p-n \\
m-n
\end{array}\right) \rho_{p, m}=\sum_{m=n}^{p} \frac{\left(\begin{array}{c}
p-n \\
m-n
\end{array}\right)}{\left(\begin{array}{c}
p \\
m
\end{array}\right)} \lambda_{p, m},
$$

and finally

(9) $\quad \mu_{n}=\sum_{m=0}^{p} \frac{m(m-1)(m-2) \cdots(m-n+1)}{p(p-1)(p-2) \cdots(p-n+1)} \lambda_{p, m}$, 
if we put

$$
\lambda_{p, m}=\left(\begin{array}{c}
p \\
m
\end{array}\right) \rho_{p, m}=\left(\begin{array}{c}
p \\
m
\end{array}\right) \Delta^{p-m} \mu_{m} .
$$

The right side of equation (9) may be written as a Stieltjes integral if we introduce with Hausdorff the function $\chi_{p}(t)$ defined as follows: $\chi_{p}(t)$ is a step-function defined for $0 \leqq t \leqq 1$, with $\chi_{p}(0)=0$, its points of discontinuity being for the values $t=m / p$ $(m=0,1,2, \cdots, p)$, where its jump has the value $\lambda_{p, m}$. This function will be completely defined by the additional condition $2 \chi_{p}(t)=\chi_{p}(t+0)+\chi_{p}(t-0)$ for $0<t<1$. From the first equation (7) we get $\chi_{p}(1)=\mu_{0}$ and the conditions (8) show that $\chi_{p}(t)$ is a non-decreasing function. From (9) we obtain

$$
\begin{gathered}
\mu_{n}=\int_{0}^{1} \frac{t\left(t-\frac{1}{p}\right)\left(t-\frac{2}{p}\right) \cdots\left(t-\frac{n-1}{p}\right)}{\left(1-\frac{1}{p}\right)\left(1-\frac{2}{p}\right) \cdots\left(1-\frac{n-1}{p}\right)} d \chi_{p}(t) \\
=\int_{0}^{1} t^{n} d \chi_{p}(t)+O\left(\frac{1}{p}\right) .
\end{gathered}
$$

The remainder of the proof is similar to Hausdorff's. A theorem of E. Helly* insures the existence of a sub-sequence $\chi_{q}(t)$ which converges to a monotonic function $\bar{\chi}(t)$. When $p=q \rightarrow \infty$, equation (10) reduces to (1) with $\chi(t)=\bar{\chi}(t)$. The relation $\int_{0}^{1} t^{n} d \chi_{q}(t) \rightarrow \int_{0}^{1} t^{n} d \bar{\chi}(t)$ follows from a theorem of Helly (loc. cit. pp. 288-289) or just as well from a known theorem on Riemann in tegrals as follows:

$$
\begin{aligned}
\int_{0}^{1} t^{n} d \chi_{q}(t) & =\chi_{q}(1)-n \int_{0}^{1} \chi_{q}(t) t^{n-1} d t \\
& \rightarrow \bar{\chi}(1)-n \int_{0}^{1} \bar{\chi}(t) t^{n-1} d t=\int_{0}^{1} t^{n} d \bar{\chi}(t) .
\end{aligned}
$$

This proves the first part of the theorem. Using the fact that $\chi(t)$ is uniquely defined at all its points of continuity by the set (1) and the condition $\chi(0)=0$, Hausdorff proved (loc. cit., pp. 225226) that the whole sequence $\chi_{p}(t)$ converges to a monotonic function $\chi(t)$ which solves the problem of moments.

* E. Helly, Über lineare Funktionaloperationen, Sitzungsberichte der Wiener Akademie, vol. $121 \mathrm{IIa}$ (1912), p. 286. 
The second statement of the theorem concerning the solution of bounded variation is also readily proved. If we suppose the function $\chi(t)$ of equations (1) to be of bounded variation, then $\chi(t)=\chi^{1}(t)-\chi^{2}(t)$, with $\chi^{1}(t)$ and $\chi^{2}(t)$ both non-decreasing functions. In Hausdorff's notation (loc. cit., p. 231) we have $\lambda_{p, m}=\lambda_{p, m}{ }^{1}-\lambda_{p, m}{ }^{2}$ and hence

$$
\sum_{m=0}^{p}\left|\lambda_{p, m}\right| \leqq \sum_{m=0}^{p} \lambda_{p, m}^{1}+\sum_{m=0}^{p} \lambda_{p, m}^{2}=\mu_{0}^{1}+\mu_{0}^{2},
$$

which proves the necessity of the condition (3). Conversely, if (3) or $\sum_{m=0}^{p}\left|\lambda_{p, m}\right|<K$ holds for $p \rightarrow \infty$, then the sequence $\chi_{p}(t)$ is a sequence of uniformly bounded functions of uniformly bounded variations. Another theorem of E. Helly* insures the existence of a sub-sequence $\chi_{q}(t)$ converging to a function $\bar{\chi}(t)$ of bounded variation. Just as above, on making $p=q \rightarrow \infty$, equation (10) becomes (1) with $\chi(t)=\bar{\chi}(t)$ and the second part of the theorem is proved. Moreover, formula (10) and the same arguments used by Hausdorff for the previous case permit us to prove that the whole sequence $\chi_{p}(t)$ converges to a function $\chi(t)$ of bounded variation satisfying the equations (1).

\section{The University of Chicago}

* E. Helly, loc. cit., $\S 6$, Theorem 7 on p. 283 . This theorem is a corollary of his first theorem used above. 